\title{
The Coordination of Production and Delivery Problem with Available Constraint and Rejection
}

\author{
Xie Xie ${ }^{* 1, a}$, Xiangyu Kong ${ }^{2, b}$ and Yongyue Zheng ${ }^{3, c}$ \\ ${ }^{12}$ Key Laboratory of Manufacturing Industrial and Integrated Automation, Shenyang University, \\ China \\ ${ }^{3}$ Liaoning Institute of standardization, Shenyang, China

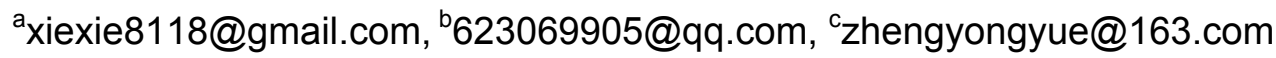

Keywords: Scheduling, Unavailability interval, Rejection, Heuristic.

\begin{abstract}
In this paper we considered a scheduling problem including both production and job delivery, with machine availability and job rejection considerations. The objective is to minimize the arrival time of the last delivery batch of accepted jobs to the distribution center plus the total penalties of rejected jobs. Based on this NP-hard problem, we propose a heuristic with a worst-case error bound of 6 , and the algorithm running time is $O(n \log n)$.
\end{abstract}

\section{Introduction}

Scheduling jobs on single machine is a classical problem that has been widely studied for more than five decades, but at the most situations, each machine is available over the production period, and all the jobs can be accepted and processed on machines, however, due to the deterministic or random factors, a machine may not usually produce, at the same time some producers in order to obtain more and more profits, they will reject these jobs which need long producing time. Moreover, in recent years, supply chain management is more important, it made up of all the stages of value creation such as supply, production, and distribution at which value is added to a manufactured and delivered product. So we put the unavailable interval and rejection into the coordination of both production and delivery scheduling problem, obviously, it is highly study value.

The machine scheduling problem with rejection was first introduced by Bartal et al. [1] and the objective is to minimize the sum of the makespan for the accepted jobs and the total rejection penalties of the rejected jobs on multi-processor scheduling problem. Numerous references studied the similar problems, they did not only propose a heuristic, but also give a full polynomial time approximation scheme, such as Zhang et al. [2] considered the single machine scheduling with release dates and rejection to minimize the sum of makespan of accepted jobs and total penalties of the rejected jobs, they proposed a heuristic, and proved the worst case is 2. Kacem and Mahjoub [3] considered a single machine scheduling problem with a deterministic non-availability interval, and the objective is to minimize the completion time of jobs, they attempted to propose a full polynomial time approximation scheme(FPTAS), and the running time is $O\left(\varepsilon^{2} / n^{2}\right)$. Kacem and Kellerer [4] studied single machine scheduling problem with releases dates, unavailability interval and rejection, they propose a 2-approximation algorithm to solve this problem. He et al. [5] considered a class of the two-stage scheduling problems that are production and the delivery, for the process environment all jobs processed on only on machine, and delivery by only one vehicle to one customer, the objective is to minimize the arrival time that all jobs finished and delivered to the customers and the vehicle returns to processing center, they proposed a approximation algorithm with the worst case is $5 / 3$. Chang and Lee. [6] considered the problem of the coordination of produce and delivery where each finished job has a different size; hence, assigning jobs to delivery batches amounts to solving the bin-packing problem. They proposed a heuristic with a worst-case analysis for each special scenario associated with different processing settings and costumers. Hall and Potts [7] considered a variety of scheduling, batching, and delivery scenarios that arise in an supply chain, the objective is to 
minimize the overall scheduling and delivery cost, for the problem is vary complexity, they proposed a heuristic. Wang and Cheng [8] considered both job production and distribution, at the same time with an unavailability interval, only one vehicle is available to transport jobs, and the vehicle can load at most $k$ jobs, the objective is to minimize the arrival time of the last delivery batch to distribution center, further they studied three scenarios of the problem, respectively processes heuristics to solve them, and proved the worst-case.

In this paper, we considered a scheduling problem, which include both production and job distribution, at the same time with machine availability constrain and each job can be rejected. The whole transport process has only one vehicle, and the vehicle capacity constrain, at most load $K$ jobs, the manufacturing settings is a single machine. In production period, there exists an unavailable interval on a machine for processing jobs due to preventive maintenance, and each job may be rejected. The objective is to minimize the arrival time of the last delivery batch of accepted jobs to the distribution center plus the total penalties of rejected jobs.

\section{Problem formulation}

Our problem is formally stated as follows: given a set of $n$ jobs $N=\left\{J_{1}, J_{2}, \ldots, J_{n}\right\}$. All accepted jobs are first produce on single machine, then delivery to distribution center. In the producing period exist two subjects: subject (1) manufacturers consider their benefits, they can reject some job that have long producing time and little penalty; subject (2) the machine have a determinate unavailability interval for maintain. We assume that all the parameters are positive integer:

$p_{j}$ : the processing time for job $J_{j}$;

$w_{j}$ : the reject penalty for job $J_{j}$;

$\left[T_{1}, T_{2}\right]$ : an unavailability interval, in this interval the machine should not be processed each job;

$\sigma:$ a feasible schedule for the set of $N$, means that jobs sequence;

$B_{k}$ : the kth delivery batch;

$\alpha\left(B_{k}\right)$ : the completion time of processing the last job of $B_{k}$ in $\sigma$;

$C\left(B_{k}\right)$ : the vehicle returning time to the producing center for batch $B_{k}$;

$q_{1}$ and $q_{2}: n=K q_{1}+q_{2}, q_{1}$ and $q_{2}$ are positive integers, $0 \leq q_{2} \leq K$;

$\varphi=\left[B_{1}, B_{2}, \ldots, B_{m}\right]:$ a transport scheme and $q_{1}+1 \leq m \leq n$;

$(\sigma, \varphi)$ : a solution of the problem;

$C(\sigma, \varphi)$ : the completion time that the last batch jobs for $(\sigma, \varphi)$ arrive at distribution center and the vehicle return producing center;

$C^{*}(\sigma, \varphi)$ : the optimal completion time that the last bath jobs arrive at distribution center and the vehicle return producing center;

$f^{H}(\sigma, \varphi)$ : objective value by heuristic algorithm $H$ solve the problem, $f^{H}(\sigma, \varphi)=C^{H}(\sigma, \varphi)+\sum_{j \in R^{\prime}} w_{j}$;

$f^{*}(\sigma, \varphi)$ : the optimal value for this problem $f^{*}(\sigma, \varphi)=C^{*}(\sigma, \varphi)+\sum_{j \in R^{*}} w_{j}$.

Before resolve the problem, we will propose some assumptions: Assumption (1): the job $J_{j}$ produce is non-resumable, means that if job $J_{j}$ has not finished before $T_{1}$, is must restart at time $T_{2}$; Assumption (2): transportation time from the producing center to the distribution center is $T_{1}^{\prime}$, and the transportation time from the distribution center to the producing center is $T_{2}^{\prime}, T_{1}^{\prime}=T_{2}^{\prime}$; Assumption (3): do not consider jobs size. We denote our problem is $1 \rightarrow D \mid h_{1}$, reject $\mid C_{\max }+\sum_{j \in R} w_{j}$, denote $1 \rightarrow D$ is the coordination of jobs production and the jobs delivery with single machine; 
denote $h_{1}$ is a unavailability interval; denote reject is that all jobs may be rejected; $C_{\max }$ is the maximal time that the last delivery batch arrive at distribution center and the vehicle return to producing center; $\sum_{j \in R} w_{j}$ the sum of penalties of a set of jobs $R$, the objective function is $f=C_{\max }+\sum_{j \in R} w_{j}$.

\section{Heuristic Algorithm and Its Worst Case Analysis}

In order to obtain a approximate value by faster, we will propose some properties:

1. The jobs should be processed as early as possible, have not be exist idle time;

2. The first complete job should first transport;

3. When have $K$ jobs have been finished should be transported to distribution center immediately.

Since Wang and Cheng. [9] have been proved the optimal value of problem $1 \rightarrow D\left|h_{1}\right| C_{\max }$ satisfied the following properties, obviously, our problem $1 \rightarrow D \mid h_{1}$, reject $\mid C_{\max }+\sum_{j \in R} w_{j}$ should be satisfied the following properties:

4. For the feasible scheme of all accepted jobs, if have $\varphi(\sigma)=\left\{\varphi=\left[B_{1}, B_{2}, \ldots, B_{m}\right] \mid \bigcup_{i=1}^{m} B_{i}=A\right\}$, $A$ is the a set of accepted jobs, and $q_{1}+1 \leq m \leq|A|, \varphi=\left[B_{1}, B_{2}, \ldots, B_{m}\right]$ belong to the optimal scheme, must satisfied $\alpha\left(B_{1}\right)<\alpha\left(B_{2}\right)<\ldots<\alpha\left(B_{m}\right)$, according the delivery scheme to produce, the first transport the first produce;

5. For an optimal scheduling scheme, the full batch rule must be best scheme, $q_{1}+1=m$ and $\left|B_{1}\right|=q_{2},\left|B_{2}\right|=\ldots=\left|B_{q+1}\right|=K$;

\section{We have heuristic algorithm $H$ :}

Step 1. Sequence the jobs in SPT rule, such as accord the processing time nondecreasing order of $p_{j}$, denote the feasible schedule as $\sigma_{1}$;

Step 2. Chouse job $J_{k}$, and satisfied $p_{k}=\max \left\{p_{j} \mid J_{j} \in N\right\}$, if have $p_{k} \leq T_{1}$, sequence job $J_{k}$ before the unavailable interval, then denote the feasible schedule as $\sigma_{2}$;

Step 3. For the schedule $\sigma_{1}$, if job $J_{j}$ satisfied $p_{j} \geq w_{j}$, should reject producing, otherwise satisfied $p_{j}<w_{j}$, should be accepted, then denote the resulting schedule is $\sigma_{1}^{\prime}$;

Step 4. For the schedule $\sigma_{2}$, if job $J_{j}$ satisfied $p_{j} \geq w_{j}$, we should reject producing, otherwise satisfied $p_{j}<w_{j}$, should be accepted, then denote the resulting schedule is $\sigma_{2}{ }^{\prime}$;

Step 5. According properties 5 and 6, determine two transport schemes by step 1 and step 2, denote the transport scheme are $\varphi_{1}, \varphi_{2}$;

Step 6. According properties 5 and 6, determine two transport schemes by step 3 and step 4, denote the transport scheme are $\varphi_{1}^{\prime}, \varphi_{2}^{\prime}$;

Step 7. Let $f^{H^{2}}=\min \left\{C^{A}\left(\sigma_{1}^{\prime}, \varphi_{1}^{\prime}\right)+\sum_{j \in R} w_{j}, C^{A}\left(\sigma_{2}^{\prime}, \varphi_{2}^{\prime}\right)+\sum_{j \in R} w_{j}\right\}$, and denote $C^{A}\left(\sigma_{1}^{\prime}, \varphi_{1}^{\prime}\right)$ is maximal completion time of the accepted jobs by step 1 and step 3 , denote $C^{A}\left(\sigma_{2}{ }^{\prime}, \varphi_{2}^{\prime}\right)$ is the maximal completion time of the accepted jobs by step2 and step 4.

Since step 1 of the heuristic algorithm $H$ with running time is $O(n \log n)$, and step 1 is the most important one, so the heuristic algorithm $H$ running time is $O(n \log n)$.

Theorem 1. For problem $1 \rightarrow D \mid h_{1}$, reject $\mid C_{\max }+\sum_{j \in R} w_{j}$, have $f^{H} \leq 6 f^{*}$. 
Proof. Since the heuristic algorithm $H$ is improved to literature [8], and they have been proved the objective value and the optimal value exist a reality problem $1 \rightarrow D\left|h_{1}\right| C_{\max }$ is $C(\sigma, \varphi) \leq 3 / 2 C^{*}(\sigma, \varphi)$, we have: $w_{j} \leq p_{j}$,

$$
\begin{aligned}
f^{H} & \leq C(\sigma, \varphi) \leq 3 / 2 C^{*}(\sigma, \varphi) \leq 3 / 2\left(\sum p_{j}+n T_{1}^{\prime}+n T_{2}^{\prime}\right) \\
& \leq 3 / 2\left(\sum_{j \in A} p_{j}+\sum_{j \in R} p_{j}+n T_{1}^{\prime}+n T_{2}^{\prime}\right) \\
& \leq 3 / 2\left(\sum_{j \in A} p_{j}+\sum_{j \in R} \max \left\{p_{j}, w_{j}\right\}+n T_{1}^{\prime}+n T_{2}^{\prime}\right) \\
& \leq 3 / 2\left(4 f^{*}\right)=6 f^{*}
\end{aligned}
$$

\section{Conclusions}

In this paper, we consider the coordination of jobs production and jobs delivery, and all accepted jobs should produce on single machine with rejection and a fix unavailability interval, the problem denote $1 \rightarrow D \mid h_{1}$, reject $\mid C_{\max }+\sum_{j \in R} w_{j}$. The objective is to minimize the accepted jobs makespan plus the sum of rejected jobs penalties. Since the problem is NP-hard, so we provided a heuristic algorithm in order to obtain an approximate value, and proved the worst case is 6 with the running time $O(n \log n)$.

\section{Acknowledgements}

This research is supported by National Natural Science Foundation of China (Grant No. 71201104). The colleges and universities of Liaoning Province outstanding talent support plan (LJQ2014133).

\section{References}

[1] Y. Bartal, S. Leonardi, A.M. Spaccamela, J. Sgall, L. Stougie, Multi-processor scheduling with rejection, SIAM Journal on Discrete Mathematics, 13 (2000), 64-78.

[2] L.Q. Zhang, L. Lu, J.J. Yuan, Single machine scheduling with release dates and rejection, European Journal of Operation Research, 198 (2009), 975-978.

[3] I. Kacem, A.R. Mahjoub, Fully polynomial time approximation scheme for the weighted flow-time minimization on a single machine with a fixed non-availability interval, Computers and Industrial Engineering, 56 (2009), 1708-1712.

[4] I. Kacem, H. Kellerer, Fast approximation algorithms to minimize a special weighted flow-time criterion on a single machine with a non-availability interval and release dates, Journal of Scheduling, 14 (2011), 257-265.

[5] Y. He,W. Zhong, H. Gu, Improved algorithms for two single machine scheduling problems, Theoretical Computer Science, 363 (2006), 257-265.

[6] Y.C. Chang and C.Y. Lee, Machine scheduling with job delivery coordination, European Journal of Operation Research, 158 (2004), 470-487.

[7] N.G. Hall and C.N. Potts, Supply chain scheduling: Batch and delivery, Operations Research, 51 (2003), 566-583. 
[8] X.L. Wang, TC. Edwin Cheng. Machine scheduling with an availability constraint and job delivery coordination, Naval Research Logistics, 54 (2007), 11-20. 